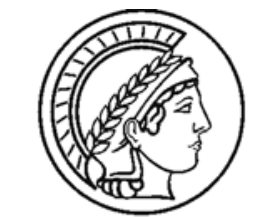

$\overline{\text { MAX-PLANCK-GESELLSCHAFT }}$

Z. Anorg. Allg. Chem. 634 (2008) 2014

\title{
Hydrotalcit-artige (Cu,Zn,Al)-Hydroxykarbonate als Vorstufen für Kupferkatalysatoren in der Methanolchemie
}

\author{
M. Behrens, S. Kühl \\ Fritz Haber Institute of the Max Planck Society, Department of Inorganic Chemistry, \\ Faradayweg 4-6, D-14195 Berlin, Germany
}

* Corresponding author: e-mail behrens@fhi-berlin.mpg.de,

Published Online: 28 Aug 2008

Keywords: Kupfer $•$ Schichtverbindungen $\bullet$ Heterogene Katalyse $\bullet$ Methanolsynthese $\bullet$ Methanoldampfreformierung

Methanol ist aufgrund seiner einfachen Handhabbarkeit ein attraktiver Wasserstoffträger für mobile Anwendungen von Brennstoffzellen. Mit der industriell bedeutsamen Methanol-synthesereaktion aus $\mathrm{H}_{2}$ und $\mathrm{CO}_{2}$ und der formalen Rückreaktion am Ort der Brennstoffzelle, der Dampfreformierung von Methanol, steht ein $\mathrm{CO}_{2}$-neutrales und damit nachhaltiges Reaktionssystem zur Verfügung. Kupferbasierte Katalysatoren sind in beiden Reaktionen aktiv. Industriell werden für die Methanolsynthese $\mathrm{Cu} / \mathrm{ZnO} / \mathrm{Al}_{2} \mathrm{O}_{3}$ Katalysatoren eingesetzt.

Typische Vorstufenverbindungen für die Herstellung dieser Katalysatoren sind gemischte Cu,Zn,(Al)-Hydroxykarbonate. Industriell hergestellte Vorstufen sind oft heterogen und enthalten eine Hydrotalcit-artige Phase der formalen Zusammensetzung $(\mathrm{Cu}, \mathrm{Zn})_{1-\mathrm{x}} \mathrm{Al}_{\mathrm{x}}(\mathrm{OH})_{2}\left(\mathrm{CO}_{3}\right)_{\mathrm{x} / 2} \cdot \mathrm{m} \mathrm{H}_{2} \mathrm{O}$. Wir konnten durch ein Co-Fällungsprozeß eine Cu-reiche Form $(\mathrm{Cu} / \mathrm{Zn}=7: 3)$ dieser Verbindung phasenrein herstellen. Die strukturellen und spektroskopischen Eigenschaften einer Probenserie mit $0,3<\mathrm{x}<0,4$ sowie die thermische Zersetzung zu den Oxiden und die anschließende Reduktion der CuO-Komponente zu Herstellung von aktiven $\mathrm{Cu} / \mathrm{ZnO} / \mathrm{Al}_{2} \mathrm{O}_{3}$-Katalysatoren wurden untersucht. Elektronen-mikroskopische Untersuchungen zeigen, dass sich aus den einphasigen Vorstufen Katalysatoren mit homogener Mikrostruktur herstellen lassen. Die entstandenen Kupferpartikel sind kugelförmig und mit Durchmessern um $5 \mathrm{~nm}$ sehr klein. Typischerweise sind sie zu einem großen Teil in einer amorphen Matrix aus $\mathrm{ZnO} / \mathrm{Al}_{2} \mathrm{O}_{3}$ oder $\mathrm{ZnAl}_{2} \mathrm{O}_{4}$ eingeschlossen (Abb).
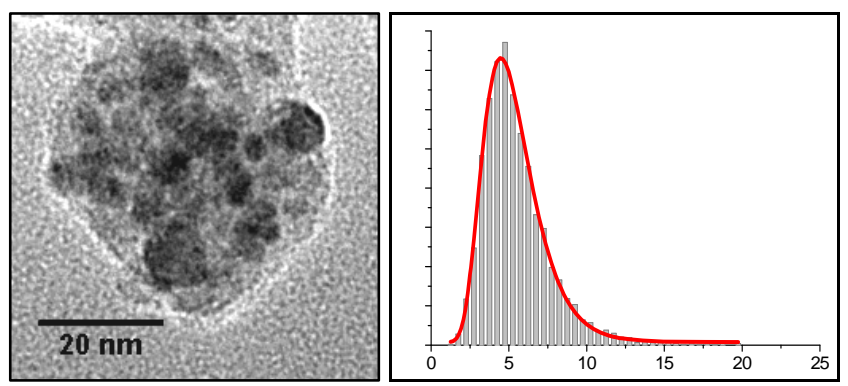

Typische TEM-Aufnahme und Größenverteilung der Cu-Partikel eines ex-Hydrotalcit-Cu/ZnO/Al $\mathrm{O}_{3}$-Katalysators

In unserem Beitrag werden die Ergebnisse dieser Untersuchungen und katalytischer Tests präsentiert, präparative Strategien zur Erhöhung der zugänglichen Kupferobenfläche, wie z.B. die Fällung aus einer Mikroemlusion, vorgestellt und die Rolle der Hydrotalcit-artigen Vorstufen in der industriell relevanten Katalysatorpräparation diskutiert. 